\title{
Raman scattering properties of structural defects in SiC
}

\author{
Xianfeng Feng ${ }^{a}$, Yuan Zang ${ }^{b}$ \\ Department of Electronic Engineering, Xi'an University of Technology, Xi'an 710048, P.R.China \\ afengxf@xaut.edu.cn, bangyuan@xaut.edu.cn
}

Keywords: SiC; defects; Raman spectra

Abstract: Although silicon carbide crystals have been commercially available, the structural discontinuity in crystal always leads to complex interplay between the atomic positions and the electronic structures, hence results in the crystal reconstructing structurally and electronically. Here we investigate the vibrational properties of $6 \mathrm{H}-\mathrm{SiC}(6 \mathrm{H}$-polytype silicon carbide) crystals containing structural defects, e.g. micropipes (MPs), screw-dislocations (SDs) and threading dislocations (TDs), by Raman scattering. For the first-order Raman scattering, the intensity of the transverse optical phonon band centered at $\sim 796 \mathrm{~cm}^{-1}$, which corresponds to the phonon mode at the $\Gamma$ point in $3 \mathrm{C}$-SiC (3C-polytype silicon carbide), is sensitive to these structural defects. But the second-order Raman features of the structural defects have no distinguished difference. In addition, the carrier concentration within an MP might be higher than that in a SD or a TD, revealing that the carrier traps surrounding the MP are lower than that for the SD or TD. These results offer a simple way for investigating the electrical properties of structural defects in SiC crystals.

\section{Introduction}

In contrast to silicon, silicon carbide ( $\mathrm{SiC}$ ) distinguishes itself by several essential properties for the electronic device production features. These outstanding characteristics include wide band gap, high saturation velocity of carriers, excellent electrical breakdown field, high maximum operating temperature and radioactive robustness [1-2]. Due to the existence of high-level semiconductor device technology developed for such semiconductors as silicon and gallium arsenide, the condition of successful translation of this technology to $\mathrm{SiC}$ is the commercial availability of high quality, large size $\mathrm{SiC}$ wafers. And the interest in fabricating high quality $\mathrm{SiC}$ devices is now growing. However, some structural defects [3-7] such as dislocations, stacking faults, misoriented grains and low-angle grain boundaries still remain to be a problem. Especially, it is generally considered that threading defects will deteriorate electrical characteristics of $\mathrm{SiC}$ power devices such as the breakdown voltage. Therefore, there is a great interest in understanding electric properties in the vicinity of these defects, which can be very helpful to detect and improve the materials and devices quality.

As we know, Raman spectroscopy now is becoming an increasingly common analysis method, which offers fast and contact-free measurements with easy sample preparation. It is a vibrational spectroscopy method that is based on the analysis of inelastically scattered light. Now, it has been employed to many applications in the fields of physics, chemistry, biology, and biomedicine [8-14]. Especially, it has been applied to $\mathrm{SiC}$ material for characterizations of polytypes [15, 16], stacking faults [17], stress [18] and doping [16, 19]. In addition, it also has been employed to SiC crystals for investigating the electric properties near a micropipe [20] and the stacking disorders related to strain [21] and the silicon segregation at the micropipe hollow[22]. After that, there are few papers 
focusing on this topic. In this paper, we have studied the properties of SiC in the vicinity and the center of structural defects by Raman spectroscopy. The difference of the electric properties between these defects is discussed.

\section{Experimental}

In this study, the samples to be investigated were cut from some home-made n-type $6 \mathrm{H}-\mathrm{SiC}$ crystals with c-plane, which were grown in the conventional sublimation method (physical vapor transport-PVT) under different conditions of seed temperature $2150-2200^{\circ} \mathrm{C}$ and growth pressure 30-40 Torr, respectively. All the under studied samples were polished both sides and etched using etchant of molten mixed $\mathrm{KOH}$ and $\mathrm{K}_{2} \mathrm{CO}_{3}$ at $400^{\circ} \mathrm{C}$ for $20-25$ minutes. The surface roughness of the unetched samples was $\sim 3 \mathrm{~nm}$ (measured by noncontact roughness tester-Taylor Surf CCI2000). The doping level of this $\mathrm{N}$-doped $6 \mathrm{H}-\mathrm{SiC}$ ingot was $\sim 10^{18} \mathrm{~cm}^{-3}$ (tested by $\mathrm{X}$-ray photoelectron spectroscope). And the micropipe defect density was for about $50 \mathrm{~cm}^{-2}$ (calculated after etching). Then Raman spectra were observed in backscattering geometry from the $<0001>$ direction at room temperature using the $\mathrm{Ar}^{+}$laser of a $25 \mathrm{~mW}$ at $514 \mathrm{~nm}$ for excitation (Renishaw). The size of the projecting light is $\sim 10 \mu \mathrm{m}$. All the spectral scans were performed in the range of $100 \sim 2000 \mathrm{~cm}^{-1}$. The accuracy of the frequency measurement was better than $\pm 1.0 \mathrm{~cm}^{-1}$.

\section{Results and discussion}

Based on group-theoretical analysis, it is shown that the Raman-active modes of a wurtzite structure, which has C6v symmetry, are the A1, E1, and E2 modes. The calculated Raman intensities of these folded modes in the transverse optical (FTO) and acoustic (FTA) branches are supported by the bond polarizability concept. It has been known that phonons caused by atomic motion parallel to the c-axis are named axial (longitudinal, L), perpendicular to the c-axis planar (transversal, T) having an acoustic (A) or optical (O) origin. The A1 and E1 phonon modes are split into LO and transverse optical (TO) modes [19]. This splitting originates from an additional force on the permanent dipole moment, for the longitudinal displacement only. Folded optical modes exhibited less splitting than acoustic modes. In a backscattering geometry, where the incident and collected light are parallel to the c axis of the sample, the A1 (LO), E1 (TO), and E2 phonons are expected to be seen in the Raman spectra at excitation energies which are nonresonant.

As we know, the existence of $\mathrm{SiC}$ polytypes has a major impact on the Raman spectra [15]. The one-phonon Raman spectra of $6 \mathrm{H}-, 4 \mathrm{H}-, 15 \mathrm{R}$ - and other polytypes of $\mathrm{SiC}$ can be explained by the folding of the Brillouin zone due to the polytype behavior of $\mathrm{SiC}$ [23]. On the other hand, the Raman spectra of these phonon modes, especially the folded transverse modes are also greatly affected by the structural defects such as dislocations and micropipes. Here, we will discuss the effects of these defects on the Raman spectra.

\section{Analysis of the vicinity of micropipe}

Fig. 1 shows a top view of micropipes and the corresponding vicinity in bulk $6 \mathrm{H}-\mathrm{SiC}$. The corresponding Raman spectra are shown in Fig. 2. Combined with Fig. 2, Table 1 shows the frequency of the folded transversal (FTA, FTO) and longitudinal (FLA, FLO) phonon modes for the acoustic and optical branches of the dispersion curves revealed from the Raman scattering results. 

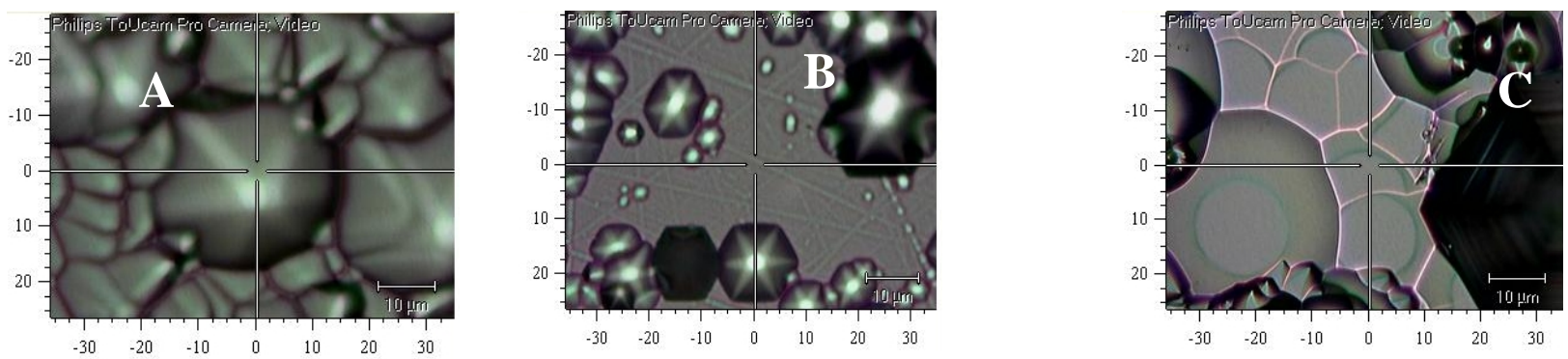

Fig. 1 Micropipe and its vicinity observed by the incidental CCD detector of Raman microscope (A) the area far from the micropipe; (B) the region away from a micropipe with a moderate distance; (C) the vicinity of a micropipe.

Table 1 Phonon modes of the vicinity of a micropipe observed by Raman spectroscopy

\begin{tabular}{ccccccc}
\hline & FTA & FTA & FLA & FTO & FTO & LOPC \\
Reference[15] & 150 & 266 & 504,514 & 766 & 787 & 968 \\
Spectrum a & 148 & 264 & 503,513 & 765 & 788 & 968 \\
& 0.33 & & & & & \\
x & $(6 \mathrm{H})$ & $1(6 \mathrm{H})$ & $0.67(6 \mathrm{H})$ & $1(6 \mathrm{H})$ & $0.33(6 \mathrm{H})$ & $0(6 \mathrm{H})$ \\
\end{tabular}

Raman spectrum $\mathrm{C}$ in Fig. 2 and its corresponding frequencies in Table 1 reveal the intense bands at $148(6 \mathrm{H}-), 264(6 \mathrm{H}-), 503(6 \mathrm{H}-), 513(6 \mathrm{H}-), 765(6 \mathrm{H}-), 788(6 \mathrm{H}-)$ and $968\left(6 \mathrm{H}^{-}\right) \mathrm{cm}^{-1}$, which indicates that there are no other polytype inclusions in this etched $6 \mathrm{H}-\mathrm{SiC}$ wafer. It also can be seen that almost all the observed peaks are shifted toward lower values, except the peaks at $788 \mathrm{~cm}^{-1}$. This phenomenon implies that this n-type wafer has been doped with shallow level impurity such as nitrogen. Fig. 3 shows local amplification of the FTO mode corresponding to Fig. 2, and the inset is the local amplification for the peaks at 503 and $513 \mathrm{~cm}^{-1}$. It can be found that the shift in the observed area is constant. This indicates a homogeneous stress in this part of the crystal. Meanwhile, the amount of plasmon damping is attributing to the spectral shape due to carrier scattering. According to Ref.[15], the increase of the carrier density will improve the value of $\omega_{\mathrm{P}}$ (plasmon frequency), and hence enhance the coupling interaction between phonons and plasmon. Meanwhile, increasing $\omega_{\mathrm{P}}$ will enlarge the dampening of phonon. Consequently, the intensity of FLO becomes lower and the full-width at half-maximum is broadened [24]. Therefore, it can be seen from Fig.3 that the carrier density near an MP (micropipe) is lower than that far from an MP, which reveals that there exists carrier trap effect near an MP. Meanwhile, we also have calculated the FWHM (full width at half maximum) of the FLO mode in the Fig.3. It has been revealed that the FWHM of Raman peak taken from the area near an MP $\left(\sim 9 \mathrm{~cm}^{-1}\right)$ is higher than that of a region departing from an MP a distance ( $\sim \mathrm{cm}^{-1}$ for 'moderate' and 'far from' case). It can also be seen that the structural defects really reduce the phonon lifetime, and hence to cause a broadening of phonon Raman bands.

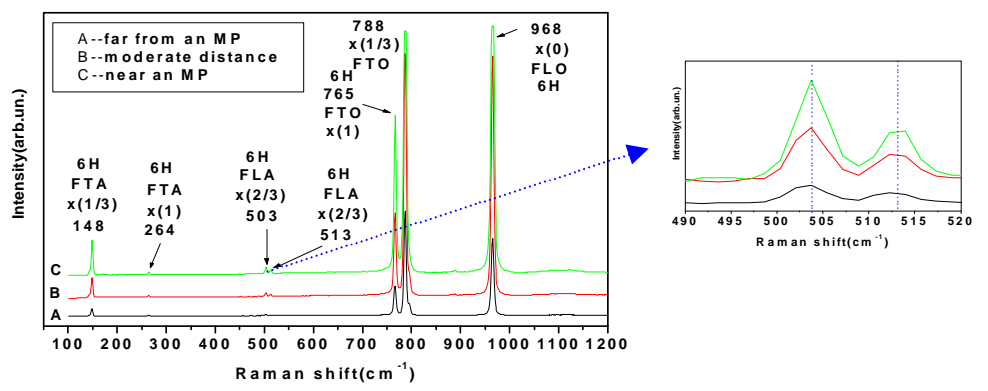

Fig. 2 The first-order Raman spectra corresponding to Fig. 1 


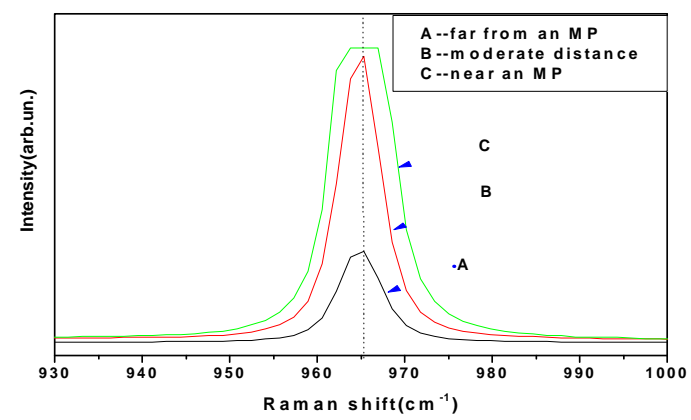

Fig. 3 Local amplification for the FLO mode (peaking at $967 \mathrm{~cm}^{-1}$ ) in Fig. 2

\section{Analysis of the center of structural defects}

Fig. 4 shows a top view of the centers for micropipe, screw-dislocation (SD) and threading edge dislocation (TED) in etched 6H-SiC. The Raman spectra corresponding to Fig. 4 are shown in Fig. 5. Fig. 6 shows local amplification of the FTO mode corresponding to Fig. 5. It can be seen that the FLO phonon frequency for an MP is shifted to higher wavenumber than those for the SD and TED. To be noted that the size of the incident light is $\sim 10 \mu \mathrm{m}$, it is larger than that of the hollow core in an MP. On the other hand, according to the above analysis, it was found that the value of $\omega \mathrm{P}$ increased when the doping concentration (n) increased, enhancing the coupling interaction between phonons and plasmon. Then the FLO phonon frequency increased and shifted to higher wavenumber when the carrier concentration was increased. It is corresponding to the phenomenon observed in Fig. 6. In other words, the carrier concentration within an MP might be higher than that in a SD or a TED. It may reveal that the carrier traps surrounding the MP are lower than that for the SD or TED. These carrier traps maybe mainly introduced by the surface states, which induce the carrier concentration of the surface distinguishing from that of the body. Meanwhile, we also calculate the FWHM of the PLO mode corresponding to Fig.6. It has been revealed that the FWHM of Raman peak taken from the center of an MP $(\sim 7 \mathrm{~cm}-1)$ is higher than that of dislocations $(\sim 6 \mathrm{~cm}-1)$. It can also be seen that the MP has a more remarkable impact on the phonon lifetime when comparing with dislocations, and hence to cause a broadening of phonon Raman bands.
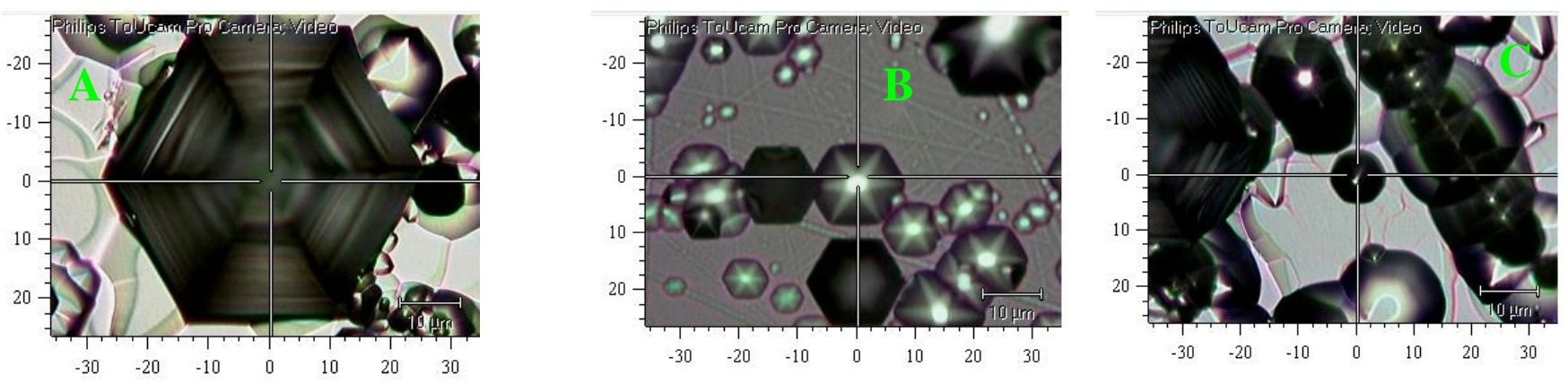

Fig. 4 Images of micropipe, screw-dislocation and threading-edge-dislocation observed by the incidental CCD detector of Raman microscope (A) the center of a micropipe; (B) the center of a screw-dislocation; (C) the center of a threading-edge-dislocation. 


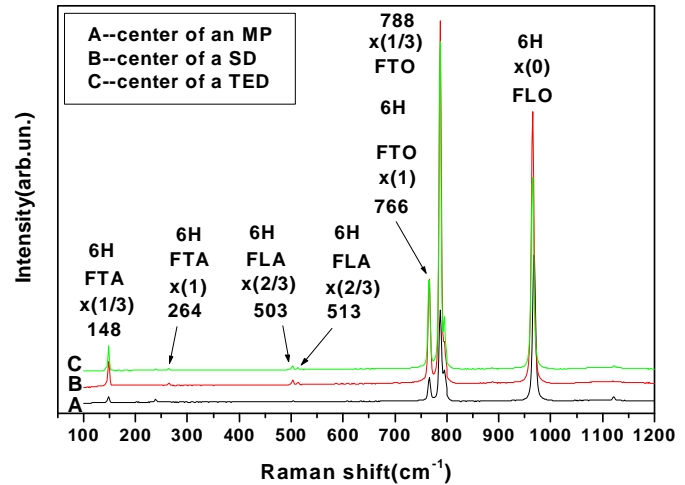

Fig. 5 The first-order Raman spectra corresponding to Fig. 4 Fig. 6 Local amplification for the FLO mode in Fig. 5

To further investigate the electrical properties of these structural defects, we focus on the observed feature peak at $796 \mathrm{~cm}-1$. Fig. 7 shows local amplification of the FTO mode corresponding to Fig. 5. It can be found that the shift in the observed area is constant. This indicates the thermal stress is released homogeneously in this part of the crystal surrounding these defects. Therefore, it is worth noting that the concomitant peak at $\sim 796 \mathrm{~cm}-1$ can be identified as feature peak not only for micropipes, but also for screw-dislocations and threading dislocations in $6 \mathrm{H}-\mathrm{SiC}$, which might be due to the structural discontinuity at the defects leads to reconstructing structurally and electronically. It also revealed that the feature peak of the TED is sharper than that of the MP and SD, which may be induced by the inhomogeneous stress within these defects. To be simple, here we could also call this FTO (0), which was similar to the one in Ref.[17] and Ref.[23]. It can be assumed that the bonding forces between $\mathrm{Si}(\mathrm{C})$ and $\mathrm{C}(\mathrm{Si})$ atomic planes are the same for hexagonal and cubic environments, then the Raman intensity of the FTO modes given by the equation in Ref. [17] can be shown as following form:

$$
W=S[n(\omega)+1] \omega^{-1}|A(q)-B(q)|^{2}|c(q)|^{2}
$$

and

$$
c(q)=N^{-2} \sum \alpha_{i} \exp \left(i q z_{i}\right)
$$

Here, $\mathrm{S}$ is a constant independent of $\omega, \mathrm{n}(\omega)$ is the Bose factor, $\mathrm{q}$ is the wave vector of phonons, and $\mathrm{A}-\mathrm{B}$ is the oscillation amplitude of the relative displacement of neighboring $\mathrm{Si}$ and $\mathrm{C}$ planes. $\mathrm{c}(\mathrm{q})$ is called the Raman structure factor and given by Fourier transform of the bond Raman polarizability $\alpha \mathrm{i}$ which is equal to the $\mathrm{xx}$, yy, and $\mathrm{xy}$ components of the bond Raman polarizability tensor. As for the FTO (0) mode $\mathrm{q}$ can be put $\mathrm{q}=0$, then the arrangement of the bond Raman polarizability along the $\mathrm{c}$ axis in a unit cell can be expressed as $\alpha=(\alpha \mathrm{i})=\mathrm{d}(1,1,1,-1,-1,-1)$ for the $6 \mathrm{H}$ polytype. Therefore, $\sum \alpha \mathrm{i}=0$. But of a structural defect (e.g. stacking faults, dislocations) is present, the $\mathrm{c}(0)=\sum \alpha \mathrm{i}$ will not be equal to zero due to the random Raman polarizability. Meanwhile, different structural defects have different effects on the phonons, which also influence the Raman structure factor and the oscillation amplitude of the relative displacement of neighboring $\mathrm{Si}$ and $\mathrm{C}$ planes. It implies that, for different structural defects, there exists a bit difference for the Raman intensity of the FTO modes at $\sim 796 \mathrm{~cm}-1$ as shown in Fig. 7. Anyway, whatever the peak at $\sim 796$ $\mathrm{cm}-1$ corresponding to micropipe or other defects, the intrinsic reason may be the stress field surrounding these defects.

In addition, it can be seen from Fig. 8 that there are no significant difference between these defects in the second-order Raman scattering, except some missing peaks labeled $\mathrm{d}$ and $\mathrm{h}$ for MP. 
These missing peaks might be induced by the reduction of the incident laser intensity at around the MP and SD, which was corresponding to the uneven surface in the inner wall of the MP and SD.

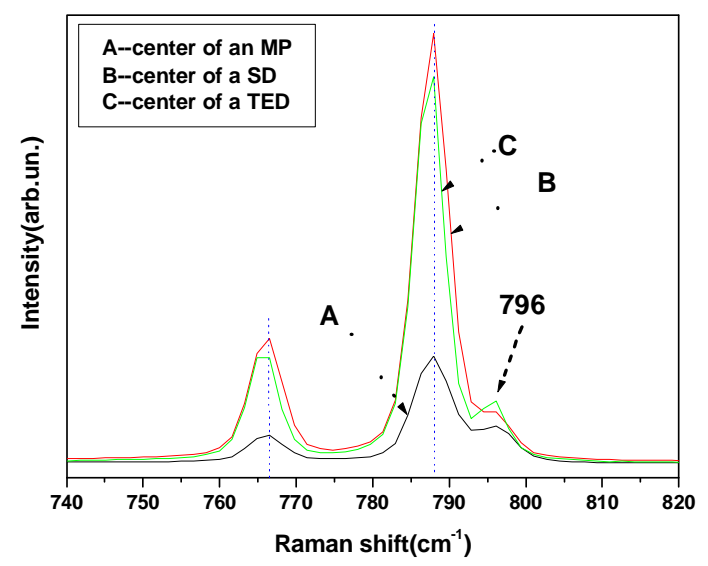

Fig. 7 Local amplification for the FTO mode in Fig. 5

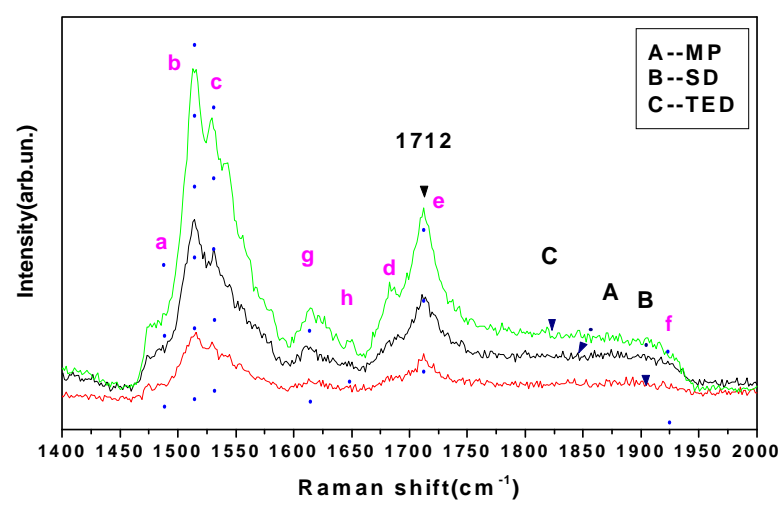

Fig. 8 Second-order Raman scattering for the bulk and the MP, SD and TED

\section{Conclusions}

In summary, the spectra of $6 \mathrm{H}-\mathrm{SiC}$ crystals containing micropipe, screw-dislocation and threading dislocation are measured using Raman scattering for the Si face. We have found that the Raman band at $\sim 796 \mathrm{~cm}^{-1}$ is sensitive to the micropipe, screw-dislocation and threading dislocation. Thus, this band can be used as a measure of the micropipe, screw-dislocation and threading dislocation. And the carrier concentration within an MP might be higher than that in a SD or a TED, revealing that the carrier traps surrounding the MP are lower than that for the SD or TED. Our work demonstrates that Raman spectroscopy is useful to characterize the structural defects such as micropipes, screw-dislocations and threading dislocations in a simple manner.

\section{Acknowledgements}

This work is financially supported by the the National Natural Science Foundation of China (Grant No. 51402230).

\section{References}

[1] L. Harris: Emis Datareviews Series, No13, Short Run Press Ltd. England 1995

[2] J.B. Casady and R.W. Johnson: Solid-State Electron Vol.39 (1996), p.1409

[3] M. Kanaya, J. Takahashi, Y. Fujiwara and A. Moritani: Appl. Phys. Lett. Vol.58 (1991), p.56

[4] K. Semmelroth, N. Schulze and G. Pensl: J. Phys. Condens. Matter. Vol.16 (2004), p.1597

[5] Z. G. Herro, B. M. Epelbaum, M. Bickermann, C. Seitz, A. Magerl and A. Winnacker: J. Cryst. Growth Vol. 275 (2005), p.496

[6] S. H. Lin, Z. H. Chen, P. Liang, Y. T. Ba and S. J. Liu: CrystEngComm Vol. 13 (2011) , p. 2709

[7] S. H. Lin, Z. H. Chen, Y. Yang, S. J. Liu, Y. T. Ba, L. B. Li and C. Yang: CrystEngComm Vol. 14 (2012), p.1588 
[8] A. Campion and P. Kambhampati: Chem. Soc. Rev. Vol. 27 (1998), p.241

[9] C. L. Haynes, C. R. Yonzon, X. Y. Zhang and R. P. Van Duyne: J.Raman Spectrosc. Vol.36 (2005), p.471

[10] M. Moskovits: J. Raman Spectrosc. Vol.36 (2005), p.485

[11] P. L. Stiles, J. A. Dieringer, N. C. Shah and R. R. Van Duyne: Annu. Rev. Anal. Chem. Vol.1 (2008), p.601

[12] J. F. Li, Y. F. Huang, Y. Ding, Z. L. Yang, S. B. Li, X. S. Zhou, F. R. Fan, W. Zhang, Z. Y. Zhou, D. Y. Wu, B. Ren, Z. L. Wang and Z. Q. Tian: Nature Vol.464 (2010), p.392

[13] Kelsey A. Stoerzinger, Julia Y. Lin and Teri W. Odom : Chem. Sci. Vol.2 (2011), p.1435

[14] G. Lu, H. Li, C. Liusman, Z. Y. Yin, S. X. Wu and H. Zhang : Chem. Sci. Vol.2 (2011), p. 1817

[15] S. Nakashima and H. Harima : phys. stat. sol. Vol.162 (1997), Vol.39

[16] S. H. Lin, Z. M. Chen, X. F. Feng, Y. Yang, L. B Li, Z. Q. Wang, P. Pan, J. Wan, H. H. Wang, Y. T. Ba, Y. Ma and Q. M. Li : Diam. Relat. Mater. Vol.20 (2011), p. 516

[17] S. Nakashima and Y. Nakatake : Appl. Phys. Lett. Vol.77 (2000), p. 3612

[18] Y. M. Lu and I. C. Leu : Thin Solid Films Vol.377-378 (2000), p. 389

[19] J. C. Burton, L. Sun, M. Pophristic, S. J. Lukacs, F. H. Long, Z. C. Feng and I. T. Ferguson : J. Appl. Phys. Vol.84 (1998), p. 6268

[20] H. Harima, T. Hosoda and S. Nakashima : Mater. Sci. Forum Vol.338-342 (2000), p. 603

[21] S. H. Seo, J. H. Park, J. S. Song, M. H. Oh : Mater. Sci. Forum Vol.457-460 (2004), p. 625

[22] E. MARTIÂN, J. JIMEÂ NEZ, M. CHAFAI : Solid State Electron. Vol.42 (1998), p. 2309

[23] D. W. Feldman, J. H. Parker, Jr., W. J. Choyke and L. Patrick : Phys. Rev. Vol.173 (1968), p. 787

[24] X. B. Li, Z. Z. Chen and E. W. Shi: Physica B Vol.405 (2010), p. 2423 